\title{
Studying the Relationship between Robustness against Mutations in Metabolic Networks and Lifestyle of Organisms
}

\author{
Sayed-Amir Marashi, ${ }^{1,2}$ Hawa Kouhestani, ${ }^{3}$ and Majid Mahdavi ${ }^{3}$ \\ ${ }^{1}$ Department of Biotechnology, College of Science, University of Tehran, Tehran 1417614411, Iran \\ ${ }^{2}$ School of Computer Science, Institute for Research in Fundamental Sciences (IPM), P.O. Box 19395-5746, Tehran, Iran \\ ${ }^{3}$ Department of Biology, Faculty of Natural Science, University of Tabriz, Tabriz 5166616471, Iran
}

Correspondence should be addressed to Sayed-Amir Marashi; marashi@ut.ac.ir and Majid Mahdavi; majid.mahdavi@tabrizu.ac.ir Received 14 August 2013; Accepted 26 September 2013

Academic Editors: A.-J. Van Dijk and C. M. Zmasek

Copyright (C) 2013 Sayed-Amir Marashi et al. This is an open access article distributed under the Creative Commons Attribution License, which permits unrestricted use, distribution, and reproduction in any medium, provided the original work is properly cited.

\begin{abstract}
Robustness is the key feature of biological networks that enables living organisms to keep their homeostatic state and to survive against external and internal perturbations. Variations in environmental conditions or nutrients and intracellular changes such as genetic mutations have the potential to change stability and efficiency of an organism. Structural robustness helps biological systems to choose alternative routes of adaptation to varying conditions. In this study, in order to estimate the structural robustness in metabolic networks we presented a novel flux balance-based approach inspired by bond percolation theory. Fourteen in silico metabolic models were studied in this work in order to examine the possible relationship between the lifestyle of organisms and their metabolic robustness. The results of this study confirm that in organisms which are highly adapted to their environment robustness to mutations may decrease compared to other organisms.
\end{abstract}

\section{Introduction}

During the last years, many researchers have studied the robustness of metabolic networks against random mutations (for a recent review, please see [1]). The purpose of these studies is to investigate the mechanisms of protection of metabolic networks against mutations and to measure the tolerance of mentioned networks against "faults" (and maybe targeted attacks).

Robustness is defined as the "insensitivity" of a system to parametric variations [2]. Variation in parameters occurs by changes in the environmental conditions or by internal alterations $[3,4]$. Structural robustness is an intrinsic property of most biological networks. Measuring the robustness of metabolic networks against mutations and gene/reaction deletion is an important question in systems biology [1]. Robustness in a metabolic network is a result of redundancy in metabolic pathways. The reason is that deficiencies in the network cannot be tolerated unless new alternative pathway(s) are evolved [5]. This is typically done by gene duplications [6] or by horizontal transfer of metabolic genes
[7]. If alternative metabolic pathways are not present in a metabolic network, for example, due to reductive evolution $[8,9]$, then the metabolic network becomes extremely fragile [10]. It has been shown that metabolic networks are exceptionally robust when compared to appropriate null models [11].

In the present work, we introduce a novel approach to the analysis of metabolic network robustness. We study the resistance of metabolic networks to deletion of reactions by removing reactions until no flux can pass through the network. We show that eukaryotes and free-living prokaryotes show much higher mutational robustness compared to organisms which are highly adapted to their habitats.

\section{Materials and Methods}

2.1. Genome-Scale Metabolic Network Models. The genomescale metabolic network models of 14 species are used in this study, including 3 eukaryotes (group 1), 6 "freeliving" prokaryotes (group 2), and 5 prokaryotes with highly 
TABLE 1: List of species used in the present work.

\begin{tabular}{|c|c|c|c|c|c|}
\hline & Species name & Specific growth conditions & $\begin{array}{c}\text { Metabolic } \\
\text { network ID }\end{array}$ & $\begin{array}{c}\text { Number of } \\
\text { reactions }\end{array}$ & $\begin{array}{l}\text { Network } \\
\text { reference }\end{array}$ \\
\hline \multirow{3}{*}{ Eukaryotes } & Saccharomyces cerevisiae & N/A & iIN800 & 1292 & {$[12]$} \\
\hline & Aspergillus nidulans & N/A & iHD666 & 711 & {$[13]$} \\
\hline & Arabidopsis thaliana & N/A & AraGEM & 672 & {$[14]$} \\
\hline \multirow{6}{*}{ "Free-living" prokaryotes } & Lactococcus lactis & N/A & - & 671 & {$[15]$} \\
\hline & Vibrio vulnificus & N/A & VvuMBEL943 & 642 & {$[16]$} \\
\hline & Escherichia coli & N/A & iAF1260 & 2167 & [17] \\
\hline & Mycobacterium tuberculosis & Human pathogen & iNJ661m & 800 & [18] \\
\hline & Methanosarcina barkeri & $\begin{array}{l}\text { Diverse anaerobic } \\
\text { conditions }\end{array}$ & iAF692 & 538 & {$[19]$} \\
\hline & Staphylococcus aureus & Human pathogen & iSB619 & 583 & [20] \\
\hline \multirow{5}{*}{$\begin{array}{l}\text { Prokaryotes with highly } \\
\text { specific growth conditions }\end{array}$} & Helicobacter pylori & $\begin{array}{l}\text { Extremely acidic conditions } \\
{[21]}\end{array}$ & iIT341 & 501 & {$[22]$} \\
\hline & Thermotoga maritima & $\begin{array}{l}\text { Extremely thermophilic } \\
\text { conditions [23] }\end{array}$ & - & 547 & {$[24]$} \\
\hline & Mycoplasma genitalium & $\begin{array}{l}\text { Intracellular conditions } \\
\text { [25] }\end{array}$ & iPS189 & 46 & {$[26]$} \\
\hline & Mycoplasma pneumoniae & $\begin{array}{c}\text { Intracellular conditions } \\
\text { [27] }\end{array}$ & iJW145 & 300 & [28] \\
\hline & Clostridium beijerinckii & $\begin{array}{l}\text { Strictly anaerobic } \\
\text { conditions [29] }\end{array}$ & iCB925 & 501 & {$[30]$} \\
\hline
\end{tabular}

specific growth conditions (group 3) [12-20, 22, 24, 26, 28, $30]$. Detailed information about the models is presented in Table 1.

2.2. Constraint-Based Analysis of Metabolic Networks. We used constraint-based analysis of metabolic networks in our study (for a brief review, please see Chapter 1 in [31]). In this modeling strategy, it is often assumed that steadystate conditions hold. Therefore, for a certain distribution of reaction fluxes, say $\mathbf{v}$, the metabolic concentrations do not change during time. In a metabolic network with $m$ metabolites and $n$ reactions, this assumption is equivalent to the following equation:

$$
\mathbf{S} \cdot \mathbf{v}=\mathbf{0}
$$

where $\mathbf{S}$ is an $m \times n$ matrix representing stoichiometric coefficients of metabolites in the reactions, $\mathbf{v}$ is the vector of the $n$ steady-state fluxes, and $\mathbf{0}$ is an $m$-dimensional zero vector. Blocked reactions [32] are those reactions which cannot carry any nonzero flux. In other words, for a blocked reaction $i$, we have $v_{i}=0$ subject to stoichiometric constraints $(\mathbf{S} \cdot \mathbf{v}=\mathbf{0})$ and reversibility constraints $\left(v_{j} \geq 0\right.$ for all irreversible reaction like $j$ ). Finding blocked reactions is typically the first step of flux coupling analysis $[32,33]$. In our study, we utilized F2C2 tool [34] for this purpose (see below).

2.3. Measuring Robustness. Our algorithm is inspired by the concept of percolation. For more information, the interested reader may refer to $[35,36]$. Here, we briefly present the main idea of the percolation theory by an example.

Figure 1(a) shows a schematic representation of the Watson-Leath experiment [37]. Suppose that we have a twodimensional steel-wire mesh (lattice). Two copper electrodes with negligible resistance are soldered to the two opposite sites of this square lattice. The resistance of the steel mesh is measured externally.

In each iteration of the experiment, a steel wire (a "bond") is cut (Watson and Leath actually studied "site" percolation; i.e., in each iteration they cut the four wires coming to a junction). The electric conductance of the lattice gradually decreases by cutting the wires. The idea is to cut steel wires randomly until no electrical current can pass through the mesh.

Let $P$ be the ratio of unblocked bonds to the total number of bonds. On average, when bonds are cut, at a critical value, say $P_{C}$, conductivity of the lattice vanishes to zero [35]. Therefore, $P_{C}$ is a random variable which can be estimated by repeating the experiment several times.

The method used in present study is based on solving a sequence of linear programming (LP) problems. In our algorithm, we used F2C2 [34] to study reactions deletions and their consequences on the activity of metabolic fluxes. The algorithm starts by correcting reversibility of reactions in a metabolic network and deleting all dead-end reactions. Then, in each iteration, one column of the stoichiometric matrix of the metabolic network (or equivalently, a reaction in metabolic network) is randomly deleted (Figure 1(b)). The procedure continues until all reactions become blocked based on the F2C2 program. Finally, the critical ratio is computed as follows:

$$
\begin{aligned}
& P_{\mathrm{C}} \\
& =\frac{\text { Number of deleted reactions }}{\text { Number of unblocked reactions in the original network }} .
\end{aligned}
$$




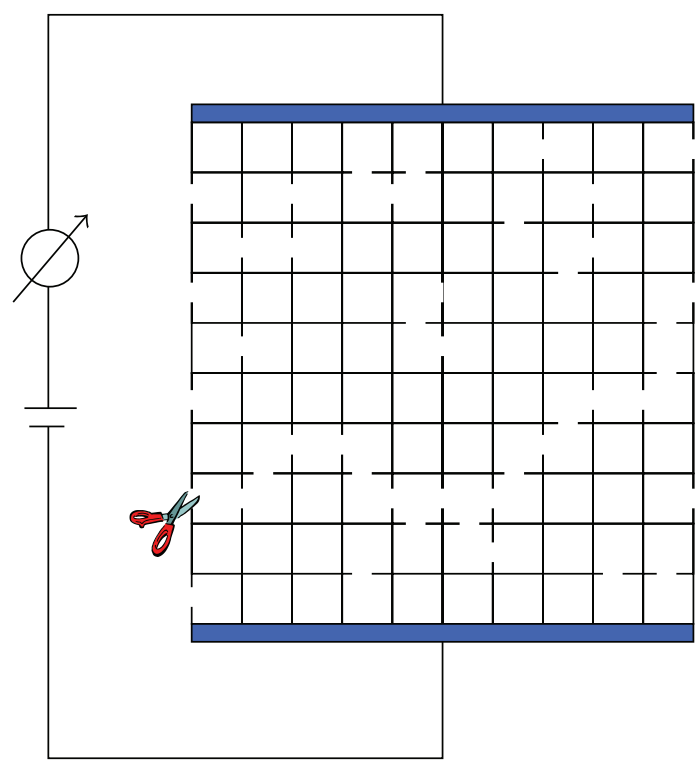

(a)

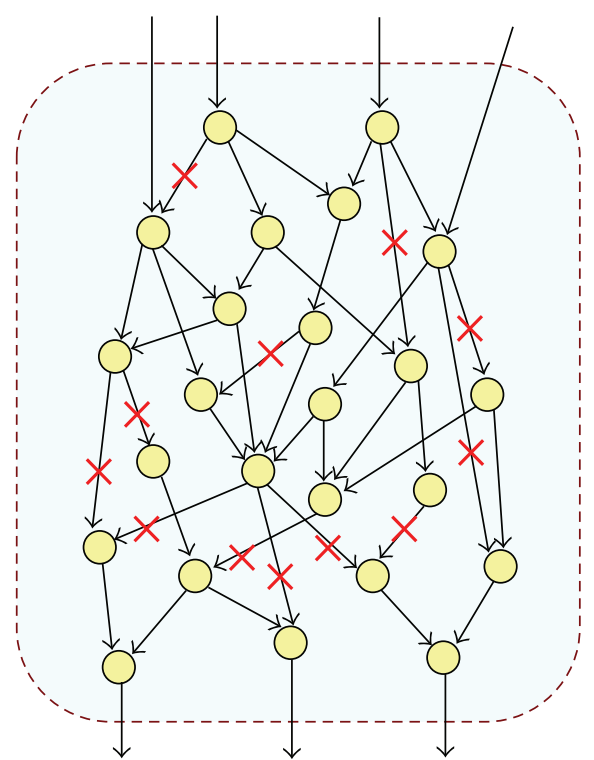

(b)

FIGURE 1: (a) Schematic representation of the two-dimensional bond percolation experiment. Conductivity of a steel-wire mesh is studied during the procedure of cutting wires (bonds) in the mesh. The goal is to find an average number of bonds which should be removed until the mesh conductivity vanishes to zero. (b) Schematic representation of our procedure. In each iteration of the modeling, a reaction is knocked out until no reaction can carry a nonzero flux in steady-state conditions.

The experiment is repeated 100 times for each network, and average $P_{C}$ values were computed for each of the metabolic network models.

We also compared our results with a classical measure of metabolic network robustness [38] based on flux balance analysis (FBA) [39]. This approach is based on in silico deletion of reactions. In each iteration, a reaction is deleted from the network and the sensitivity of the growth rate to the reaction deletion is modeled. We used the core reductive algorithm $[8,9,40]$ for this purpose. In each iteration, we find a (randomly selected) minimal reaction set which can be used to produce biomass from a minimal growth medium in steady-state conditions. In a highly robust network, a considerable number of reactions can be deleted without influencing growth, while in a sensitive network deletion of a few reactions can result in no biomass production. Therefore, the average ratio of "unnecessary" reactions to the total number of reactions can be used as a measure of network robustness. For each metabolic network, the experiment was repeated 1000 times to have a good estimation of this ratio.

2.4. Statistical Analysis. The $R$ package (http://www.rproject.org/) was used for statistical analyses. In order to compare the $P_{C}$ distributions in two organisms, one-sided two-sample $t$-test was used. To investigate the correlation between $P_{C}$ values and the number of reactions in the models, Pearson's product-moment correlation test was applied.

\section{Results and Discussion}

$P_{C}$ was defined as the critical ratio of the fluxes to be removed such that a metabolic network becomes entirely blocked

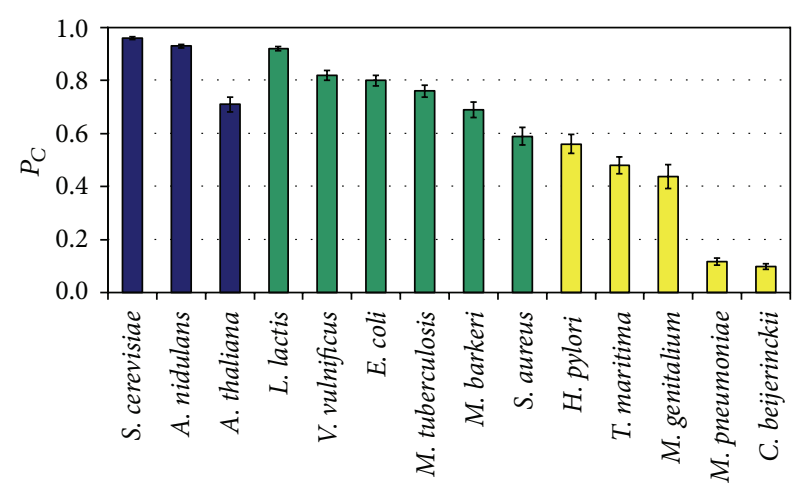

Figure 2: Average $P_{C}$ for the fourteen metabolic network models. The histograms for group 1 (eukaryotes), group 2 ("free-living" prokaryotes), and group 3 (highly-adapted prokaryotes) are shown in dark blue, green, and yellow, respectively. The error bars in this plot are the $95 \%$ confidence intervals based on one-sample $t$-test.

(Figure 1(b)). The higher the average $P_{C}$ is, the higher the number of nonessential reactions is. Thus, we chose $P_{C}$ to estimate the robustness of the metabolic networks.

Each set of deleted reactions is a cut set for the network [41] (but presumably not a minimal cut set). Therefore, the average $P_{C}$ is an estimate for the average cut set size. For each of the fourteen metabolic networks in our dataset, we computed average $P_{C}$ by repeating the reaction deletion procedure 100 times. The results of this analysis are summarized in Figure 2. From this figure, one can observe that there is comparable range of $P_{C}$ values for metabolic networks in group 1 and group 2 . However, for group 3 , we face a range of 


$\begin{array}{rcccccc} & \text { L. lactis } & \text { V. vulnificus } & \text { E. coli } & \text { M. tuberculosis } & \text { M. barkeri } & \text { S. aureus } \\ \text { S. cerevisiae } & 7.96 E-12 & 3.90 E-27 & 1.34 E-31 & 6.20 E-31 & 2.41 E-35 & 4.26 E-40 \\ \text { A. nidulans } & 1.47 E-02 & 7.29 E-22 & 6.60 E-28 & 3.65 E-27 & 1.42 E-32 & 4.42 E-38 \\ \text { A. thaliana } & 1.00 E+00 & 1.00 E+00 & 9.90 E-01 & 9.95 E-01 & 1.22 E-01 & 1.05 E-07 \\ & & & & & & \\ \text { H. pylori } & 1.58 E-37 & 2.77 E-25 & 4.47 E-16 & 9.86 E-17 & 7.21 E-08 & 1.03 E-01 \\ \text { T. maritima } & 1.24 E-50 & 1.41 E-40 & 1.06 E-29 & 1.65 E-30 & 4.06 E-18 & 2.84 E-06 \\ \text { M. genitalium } & 7.11 E-40 & 8.42 E-32 & 4.64 E-25 & 1.33 E-25 & 2.12 E-17 & 4.30 E-08 \\ \text { M. pneumoniae } & 5.26 E-150 & 4.57 E-121 & 7.08 E-94 & 8.19 E-95 & 4.64 E-74 & 1.54 E-54 \\ \text { C. beijerinckii } & 1.26 E-169 & 3.41 E-119 & 2.21 E-92 & 2.95 E-93 & 9.97 E-74 & 2.26 E-55 \\ \quad & & & & & \\ \text { Color key: } & & & & & & \\ P>10^{-2} & & & & & & \\ 10^{-2}>P>10^{-15} & & & & & & \end{array}$

Figure 3: The upper box shows the $P$ values of $t$-test for $P_{C}$ (group 1) $>P_{C}$ (group 2), while the lower box shows the $P$ values of $t$-test for $P_{C}$ (group 2) $>P_{C}$ (group 3).

$P_{C}$ values which does not overlap with the range of $P_{C}$ values for groups 1 and 2 . This observation implies that the network robustness of group 3 is much less than that of groups 1 and 2 .

To investigate the significance of differences between $P_{C}$ values of different groups, one-sided two-sample $t$-test was used. We tested whether the $P_{C}$ values of eukaryotes (group 1) are significantly greater than $P_{C}$ values of free-living prokaryotes (group 2) and whether the $P_{C}$ values of freeliving prokaryotes (group 2) are significantly greater than $P_{C}$ values of prokaryotes with highly specific growth conditions. The results are summarized in Figure 3. Obviously, the differences between group 2 and group 3 are much more significant than the differences between group 1 and group 2. This observation confirms that prokaryotes with highly specific growth conditions are significantly less robust than the free-living prokaryotes.

We also tested whether the number of unblocked reactions (and not the network structure) determines the network robustness. We found that although the correlation between the number of reactions and $P_{C}$ is positive $\left(R^{2}=0.26\right)$, it is not statistically significant $(P$ value $>0.05$ in Pearson's product-moment correlation test). Therefore, there is only a weak, if any, relationship between the number of reactions and network robustness. This finding emphasizes the importance of the network "structure" and "wiring" (as an intrinsic property in each metabolic network) in shaping the network mutational robustness.

In this study, group 1 includes eukaryotic species ( $S$. cerevisiae, $A$. nidulans, and $A$. thaliana). It is well known that $S$. cerevisiae and $A$. nidulans can adapt to a wide range of growth conditions. Moreover, A. thaliana is a multicellular organism with different tissues. For these reasons, in group 1 we expect a large number of alternative metabolic pathways, which in turn results in great robustness values. It should be noted that, in the metabolic model of A. thaliana, only 672 unblocked reactions are included. However, due to the large number of metabolic enzymes involved in the plants, this number is greatly underestimated. One expects that addition of the missing metabolic pathways to this model will greatly enhance the robustness of this network.
In group 2, we have six prokaryotes which are able to grow in different habitats. L. lactis is well known for its application to the lactic industry, while it is reported that this species is also isolated from vegetables [42] and intestinal tract of the Amur catfish [43]. On the other hand, V. vulnificus, which is a cause of deadly food poisoning and wound infections, is also present in brackish ponds [44]. Another species from this group, that is, E. coli, can easily grow in human intestine, in water, and in soil [45]. While M. tuberculosis is a human pathogen, it is proven that this bacterium has an exceptional ability to survive environmental stresses [46]. Moreover, it can grow in different growth conditions, both in vivo and in vitro [47]. S. aureus is typically known as a human pathogen. However, there is a growing body of evidence that this microorganism is also able to grow in a variety of different conditions, including foods [48] and soil [49]. M. barkeri is the only archaeon in this group, with the ability to grow in many different growth conditions ranging from rumen [50] to freshwater lagoons [51].

Group 3 includes bacterial species with highly specific growth conditions. H. pylori, a human pathogen, grows in highly acidic environment of the stomach [21], T. maritima only grows in extremely thermophilic conditions [23], and C. beijerinckii grows only in strictly anaerobic conditions [29]. M. genitalium and M. pneumoniae, on the other hand, have reduced metabolic networks which helps them to grow faster as intracellular pathogens $[25,27]$. The extreme level of adaptation in species of group 3 has resulted in the greater degree of "nonrobustness" in the metabolic networks of these organisms.

In this work, we presented a novel way of measuring metabolic network robustness in the constraint-based modeling framework. Another class of robustness measures in this framework is based on FBA [38]. We believe that our robustness measure is more suitable for comparing a variety of living organisms. The reason is that FBA is always based on a predefined objective function (e.g., biomass production flux) and a predefined growth medium (i.e., those uptake reactions which can take nonzero flux values), which can vary across species. Despite this fact, all measures of robustness 
must be correlated. For example, a highly robust metabolic network with many alternative pathways is identified as a robust network by any measure of robustness.

In order to find the relationship between our novel robustness measure and the FBA-based measure of robustness, we used our recent implementation of the corereductive algorithm [40] to obtain minimal metabolic subnetworks which are able to produce biomass. The number of reactions which can be deleted without decreasing biomass production is an FBA-based measure of network robustness. We found out that the results are qualitatively comparable, with a relatively high correlation between the two measures (Pearson's correlation $R=0.80$ ). The results confirm that our novel robustness measure is comparable with the classical measures of robustness, but with the advantage that no additional assumption is required for its computation.

\section{Acknowledgment}

Sayed-Amir Marashi is supported by a Grant from Institute for Research in Fundamental Sciences (IPM) (no. CS 1391-0$01)$.

\section{References}

[1] A. Larhlimi, S. Blachon, J. Selbig, and Z. Nikoloski, "Robustness of metabolic networks: a review of existing definitions," BioSystems, vol. 106, no. 1, pp. 1-8, 2011.

[2] M. Morohashi, A. E. Winn, M. T. Borisuk, H. Bolouri, J. Doyle, and H. Kitano, "Robustness as a measure of plausibility in models of biochemical networks," Journal of Theoretical Biology, vol. 216, no. 1, pp. 19-30, 2002.

[3] H. Kitano, "Biological robustness," Nature Reviews Genetics, vol. 5, no. 11, pp. 826-837, 2004.

[4] A. Wagner, "Distributed robustness versus redundancy as causes of mutational robustness," BioEssays, vol. 27, no. 2, pp. 176-188, 2005.

[5] T. Wilhelm, J. Behre, and S. Schuster, "Analysis of structural robustness of metabolic networks," Systems Biology, vol. 1, no. 1, pp. 114-120, 2004.

[6] L. Kuepfer, U. Sauer, and L. M. Blank, "Metabolic functions of duplicate genes in Saccharomyces cerevisiae," Genome Research, vol. 15, no. 10, pp. 1421-1430, 2005.

[7] R. A. Notebaart, P. R. Kensche, M. A. Huynen, and B. E. Dutilh, "Asymmetric relationships between proteins shape genome evolution," Genome Biology, vol. 10, no. 2, article R19, 2009.

[8] C. Pál, B. Papp, M. J. Lercher, P. Csermely, S. G. Oliver, and L. D. Hurst, "Chance and necessity in the evolution of minimal metabolic networks," Nature, vol. 440, no. 7084, pp. 667-670, 2006.

[9] K. Yizhak, T. Tuller, B. Papp, and E. Ruppin, "Metabolic modeling of endosymbiont genome reduction on a temporal scale," Molecular Systems Biology, vol. 7, article 479, 2011.

[10] G. H. Thomas, J. Zucker, S. J. Macdonald, A. Sorokin, I. Goryanin, and A. E. Douglas, "A fragile metabolic network adapted for cooperation in the symbiotic bacterium Buchnera aphidicola," BMC Systems Biology, vol. 3, article 24, 2009.

[11] A. G. Smart, L. A. N. Amaral, and J. M. Ottino, "Cascading failure and robustness in metabolic networks," Proceedings of the
National Academy of Sciences of the United States of America, vol. 105, no. 36, pp. 13223-13228, 2008.

[12] I. Nookaew, M. C. Jewett, A. Meechai et al., "The genome-scale metabolic model iIN800 of Saccharomyces cerevisiae and its validation: a scaffold to query lipid metabolism," BMC Systems Biology, vol. 2, article 71, 2008.

[13] H. David, I. Ş. Özçelik, G. Hofmann, and J. Nielsen, "Analysis of Aspergillus nidulans metabolism at the genome-scale," BMC Genomics, vol. 9, article 163, 2008.

[14] C. G. D. O. Dal'Molin, L. Quek, R. W. Palfreyman, S. M. Brumbley, and L. K. Nielsen, "AraGEM, a genome-scale reconstruction of the primary metabolic network in Arabidopsis," Plant Physiology, vol. 152, no. 2, pp. 579-589, 2010.

[15] A. P. Oliveira, J. Nielsen, and J. Förster, "Modeling Lactococcus lactis using a genome-scale flux model," BMC Microbiology, vol. 5, article 39, 2005.

[16] H. U. Kim, S. Y. Kim, H. Jeong et al., "Integrative genome-scale metabolic analysis of Vibrio vulnificus for drug targeting and discovery," Molecular Systems Biology, vol. 7, article 460, 2011.

[17] A. M. Feist, C. S. Henry, J. L. Reed et al., "A genome-scale metabolic reconstruction for Escherichia coli K-12 MG1655 that accounts for 1260 ORFs and thermodynamic information," Molecular Systems Biology, vol. 3, article 121, 2007.

[18] X. Fang, A. Wallqvist, and J. Reifman, "Development and analysis of an in vivo-compatible metabolic network of Mycobacterium tuberculosis," BMC Systems Biology, vol. 4, article 160, 2010.

[19] A. M. Feist, J. C. M. Scholten, B. Ø. Palsson, F. J. Brockman, and T. Ideker, "Modeling methanogenesis with a genome-scale metabolic reconstruction of Methanosarcina barkeri," Molecular Systems Biology, vol. 2, Article ID 2006.0004, 2006.

[20] S. A. Becker and B. Ø. Palsson, "Genome-scale reconstruction of the metabolic network in Staphylococcus aureus N315: an initial draft to the two-dimensional annotation," BMC Microbiology, vol. 5, article 8, 2005.

[21] D. R. Scott, E. A. Marcus, Y. Wen, J. Oh, and G. Sachs, "Gene expression in vivo shows that Helicobacter pylori colonizes an acidic niche on the gastric surface," Proceedings of the National Academy of Sciences of the United States of America, vol. 104, no. 17, pp. 7235-7240, 2007.

[22] I. Thiele, T. D. Vo, N. D. Price, and B. Ø. Palsson, "Expanded metabolic reconstruction of Helicobacter pylori (iIT341 GSM/GPR): an in silico genome-scale characterization of single- and double-deletion mutants," Journal of Bacteriology, vol. 187, no. 16, pp. 5818-5830, 2005.

[23] R. Huber, T. A. Langworthy, H. König et al., “Thermotoga maritima sp. nov. represents a new genus of unique extremely thermophilic eubacteria growing up to $90^{\circ} \mathrm{C}$," Archives of Microbiology, vol. 144, no. 4, pp. 324-333, 1986.

[24] Y. Zhang, I. Thiele, D. Weekes et al., "Three-dimensional structural view of the central metabolic network of Thermotoga maritima," Science, vol. 325, no. 5947, pp. 1544-1549, 2009.

[25] J. S. Jensen, J. Blom, and K. Lind, "Intracellular location of Mycoplasma genitalium in cultured vero cells as demonstrated by electron microscopy," International Journal of Experimental Pathology, vol. 75, no. 2, pp. 91-98, 1994.

[26] P. F. Suthers, M. S. Dasika, V. S. Kumar, G. Denisov, J. I. Glass, and C. D. Maranas, "Genome-scale metabolic reconstruction of Mycoplasma genitalium, iPS189," PLoS Computational Biology, vol. 5, no. 2, Article ID e1000285, 2009. 
[27] M. A. Meseguer, A. Álvarez, M. T. Rejas, C. Sánchez, J. C. PérezDíaz, and F. Baquero, "Mycoplasma pneumoniae: a reducedgenome intracellular bacterial pathogen," Infection, Genetics and Evolution, vol. 3, no. 1, pp. 47-55, 2003.

[28] J. A. H. Wodke, J. Puchalka, M. Lluch-Senar et al., "Dissecting the energy metabolism in Mycoplasma pneumoniae through genome-scale metabolic modeling," Molecular Systems Biology, vol. 9, article 653, 2013.

[29] W. J. Mitchell, "Carbohydrate uptake and utilization by Clostridium beijerinckii NCIMB 8052," Anaerobe, vol. 2, no. 6, pp. 379384, 1996.

[30] C. B. Milne, J. A. Eddy, R. Raju et al., "Metabolic network reconstruction and genome-scale model of butanol-producing strain Clostridium beijerinckii NCIMB 8052," BMC Systems Biology, vol. 5, article 130, 2011.

[31] S. A. Marashi, Constraint-Based Analysis of Substructures of Metabolic Networks, Freie Universität Berlin, Berlin, Germany, 2011.

[32] A. P. Burgard, E. V. Nikolaev, C. H. Schilling, and C. D. Maranas, "Flux coupling analysis of genome-scale metabolic network reconstructions," Genome Research, vol. 14, no. 2, pp. 301-312, 2004.

[33] L. David, S. A. Marashi, A. Larhlimi, B. Mieth, and A. Bockmayr, "FFCA: a feasibility-based method for flux coupling analysis of metabolic networks," BMC Bioinformatics, vol. 12, article 236, 2011.

[34] A. Larhlimi, L. David, J. Selbig, and A. Bockmayr, "F2C2: a fast tool for the computation of flux coupling in genome-scale metabolic networks," BMC Bioinformatics, vol. 13, article 57, 2012.

[35] A. L. Efros, Physics and Geometry of Disorder: Percolation Theory, Mir Publishers, Moscow, Russia, 1986.

[36] D. Stauffer and A. Aharony, Introduction to Percolation Theory, CRC Press, New York, NY ,USA, 1994.

[37] B. P. Watson and P. L. Leath, "Conductivity in the twodimensional-site percolation problem," Physical Review B, vol. 9, no. 11, pp. 4893-4896, 1974.

[38] J. S. Edwards and B. O. Palsson, "Robustness analysis of the Escherichia coli metabolic network," Biotechnology Progress, vol. 16, no. 6, pp. 927-939, 2000.

[39] J. D. Orth, I. Thiele, and B. O. Palsson, "What is flux balance analysis?” Nature Biotechnology, vol. 28, no. 3, pp. 245-248, 2010.

[40] S. Tabe-Bordbar and S. A. Marashi, "Finding elementary flux modes in metabolic networks based on flux balance analysis and flux coupling analysis: application to the analysis of Escherichia coli metabolism," Biotechnology Letters, vol. 35, no. 12, pp. 20392044, 2013.

[41] S. Klamt and E. D. Gilles, "Minimal cut sets in biochemical reaction networks," Bioinformatics, vol. 20, no. 2, pp. 226-234, 2004.

[42] L. Uhlman, U. Schillinger, J. R. Rupnow, and W. H. Holzapfel, "Identification and characterization of two bacteriocinproducing strains of Lactococcus lactis isolated from vegetables," International Journal of Food Microbiology, vol. 16, no. 2, pp. 141-151, 1992.

[43] H. Sugita, K. Ohta, A. Kuruma, and T. Sagesaka, "An antibacterial effect of Lactococcus lactis isolated from the intestinal tract of the Amur catfish, Silurus asotus Linnaeus," Aquaculture Research, vol. 38, no. 9, pp. 1002-1004, 2007.
[44] C. O. Tacket, T. J. Barrett, J. M. Mann, M. A. Roberts, and P. A. Blake, "Wound infections caused by Vibrio vulnificus, a marine vibrio, in inland areas of the United States," Journal of Clinical Microbiology, vol. 19, no. 2, pp. 197-199, 1984.

[45] M. A. Savageau, "Escherichia coli habitats, cell types, and molecular mechanisms of gene control," American Naturalist, vol. 122, no. 6, pp. 732-744, 1983.

[46] K. Kulka, G. Hatfull, and A. K. Ojha, "Growth of Mycobacterium tuberculosis biofilms," Journal of Visualized Experiments, no. 60, Article ID e3820, 2012.

[47] A. M. Dhople and D. L. S. Ryon, "ATP content of Mycobacterium tuberculosis grown in vivo and in vitro," Microbios, vol. 101, no. 399, pp. 81-88, 2000.

[48] M. I. Halpin-Dohnalek and E. H. Marth, "Staphylococcus aureus: production of extracellular compounds and behavior in foods: a review," Journal of Food Protection, vol. 52, pp. 267-282, 1989.

[49] K. S. Ko, S. K. Lim, S. C. Jung, J. M. Yoon, J. Y. Choi, and J. H. Song, "Sequence type 72 meticillin-resistant Staphylococcus aureus isolates from humans, raw meat and soil in South Korea," Journal of Medical Microbiology, vol. 60, no. 4, pp. 442-445, 2011.

[50] S. K. Sirohi, N. Pandey, B. Singh, and A. K. Puniya, "Rumen methanogens: a review," Indian Journal of Microbiology, vol. 50, no. 3, pp. 253-262, 2010.

[51] O. Kandler and H. Hippe, "Lack of peptidoglycan in the cell walls of Methanosarcina barkeri," Archives of Microbiology, vol. 113, no. 1-2, pp. 57-60, 1977. 

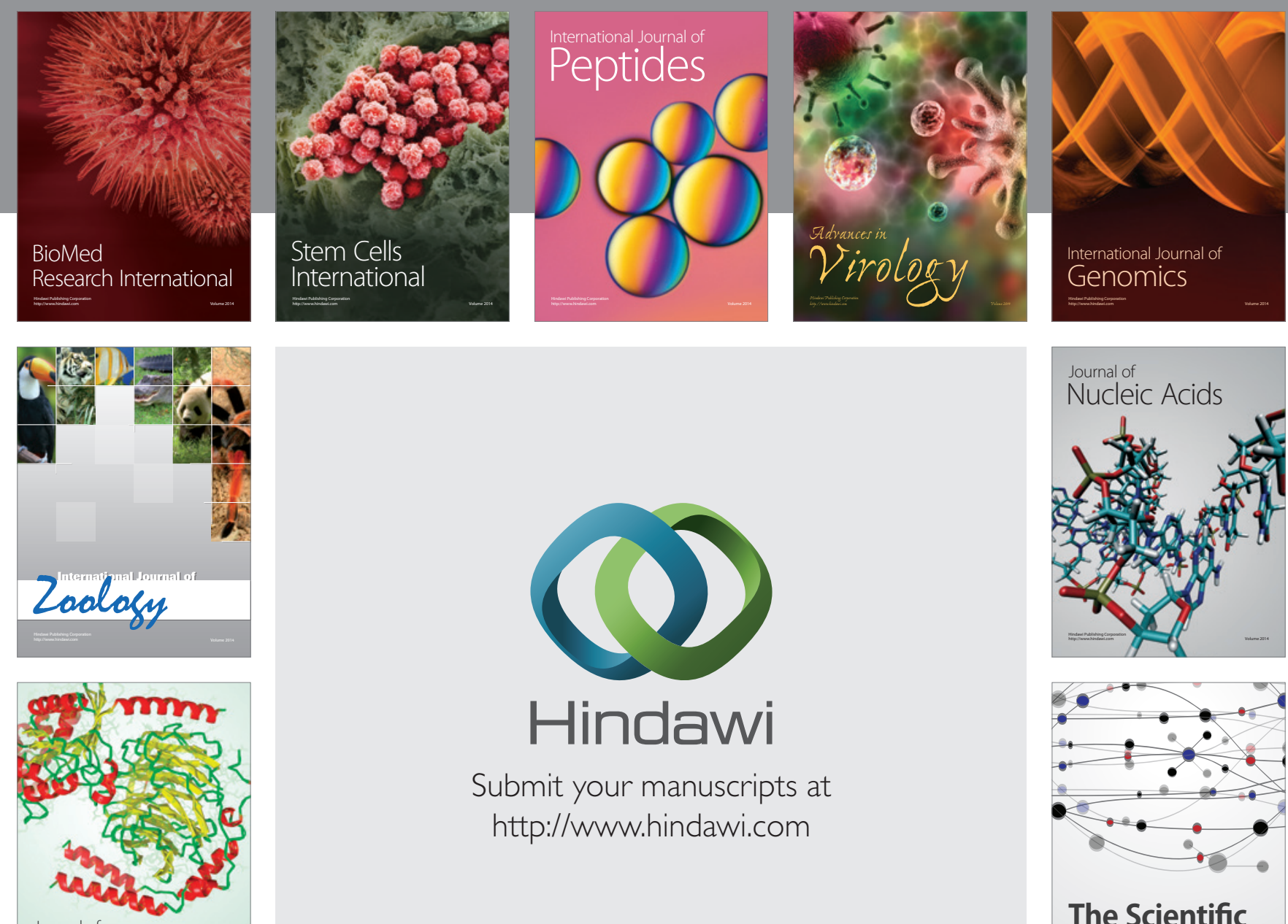

Submit your manuscripts at

http://www.hindawi.com

Journal of
Signal Transduction
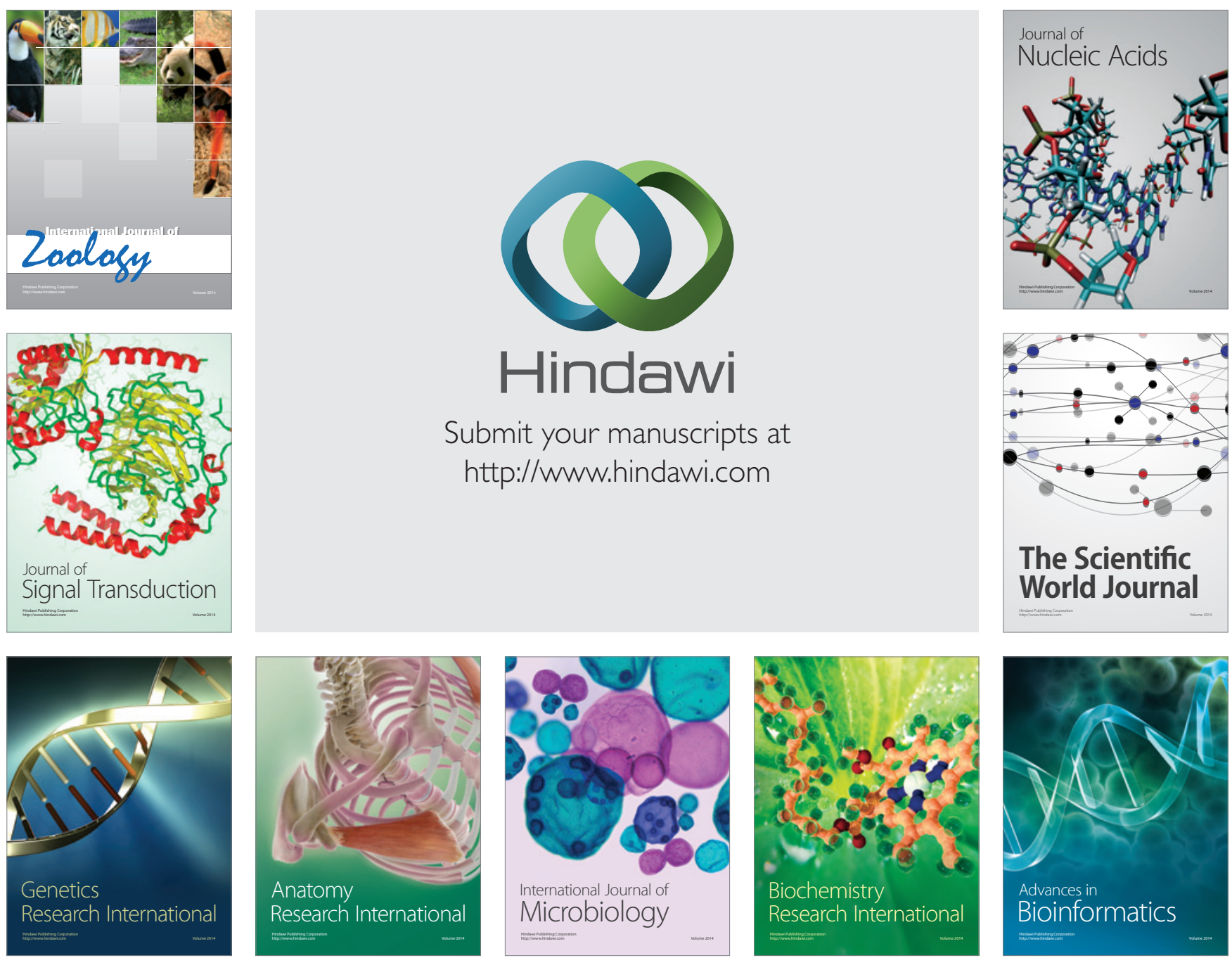

The Scientific World Journal
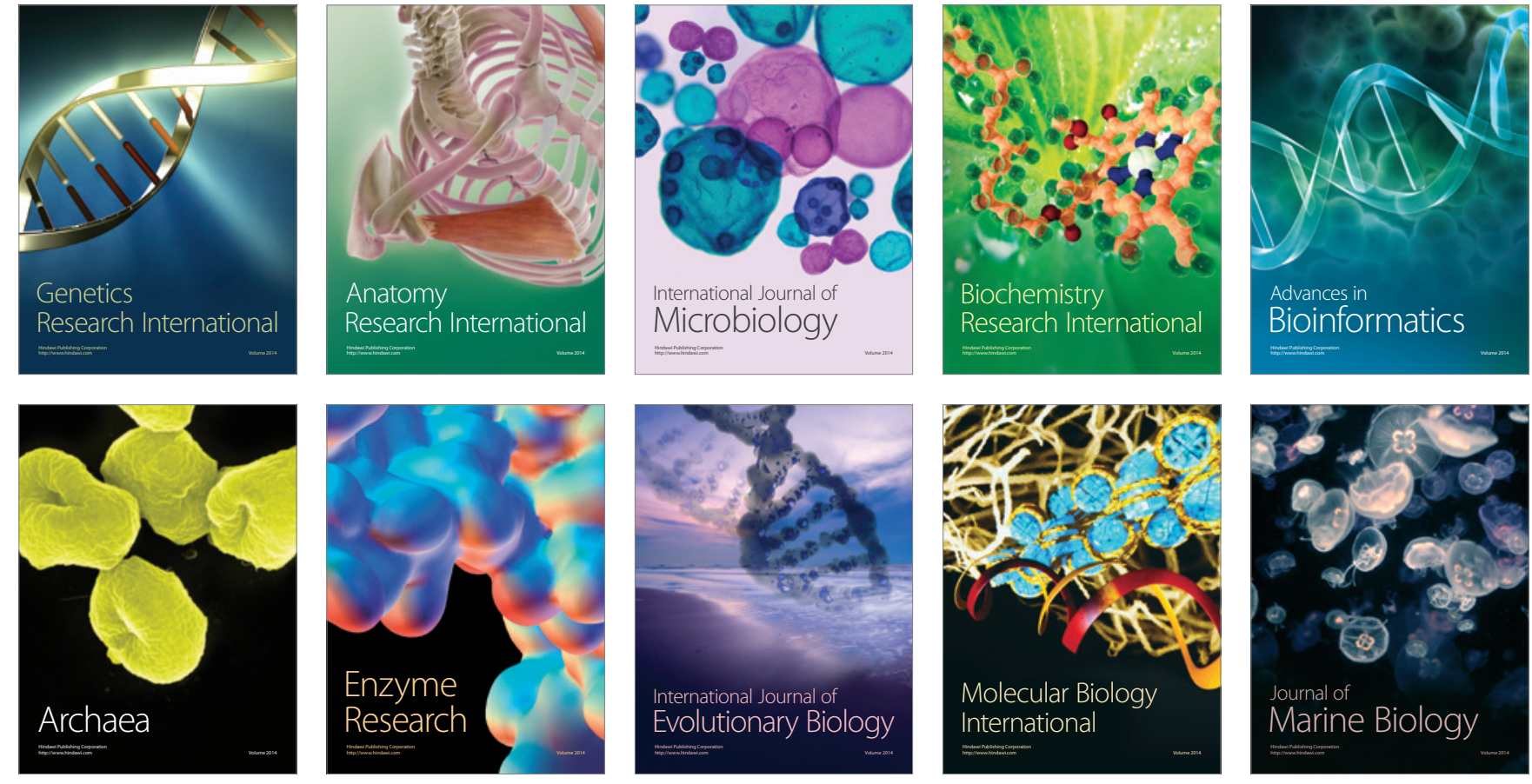